\title{
Computing a T-transitive lower approximation or opening of a proximity relation
}

\author{
L. Garmendia , A. Salvador , J. Montero \\ Complutense University of Madrid, Spain \\ Technical University of Madrid, Spain
}

\begin{abstract}
:
Since transitivity is quite often violated even by decision makers that accept transitivity in their preferences as a condition for consistency, a standard approach to deal with intransitive preference elicitations is the search for a close enough transitive preference relation, assuming that such a violation is mainly due to decision maker estimation errors. In some way, the more number of elicitations, the more probable inconsistency is. This is mostly the case within a fuzzy framework, even when the number of alternatives or object to be classified is relatively small. In this paper we propose a fast method to compute a Tindistinguishability from a reflexive and symmetric fuzzy relation, being $\mathrm{T}$ any left-continuous t-norm. The computed approximation we propose will take $\mathrm{O}\left(n^{3}\right)$ time complexity, where $\mathrm{n}$ is the number of elements under consideration, and is expected to produce a T-transitive opening. To the authors' knowledge, there are no other proposed algorithm that computes T-transitive lower approximations or openings while preserving the reflexivity and symmetry properties.
\end{abstract}

Keywords: Fuzzy relation; fuzzy proximity relation; T-transitive relation; fuzzy similarity; T-indistinguishability; T-transitive lower approximation; T-transitive opening.

\section{Introduction}

Schweizer and Sklar introduced triangular norms to generalize the triangle inequality in probabilistic metric spaces. Some common applications of tnorms are found in different intersection operators for fuzzy sets and in modeling of the logical connective AND in fuzzy logic.

The transitive property of fuzzy relations can be understood as a threshold on the degree of the relation (for example, a degree of equality) between two elements, when a degree of relation between 
those elements and a third element of a universe of discourse is known. The classical concept of transitivity is generalized in fuzzy logic by the T-transitivity property of fuzzy relations, where $\mathrm{T}$ is a triangular norm.

Consistency of a crisp preference relation has been traditionally associated to completeness together with transitivity, in such a way that decision makers are assumed to be always able to choose a best alternative from any pair of alternatives, and no contradiction follows when they are shown a sequence of preferences. Although alternative approaches can be found in the literature, a classical approach within a fuzzy context is to extend such a concept into the Ttransitivity property by means of a triangular norm $\mathrm{T}$, according to the definitions below.

Approximate reasoning applications using fuzzy inference typically use the compositional rule of inference with $\mathrm{T}$-transitive fuzzy relations such as $\mathrm{T}$ preorders, T-indistinguishabilities, similarities, or equivalence relations, allowing the comparison of objects and the formulation of complete conclusions. Algorithms that search for the T-transitivity property can be used in many approximate reasoning applications, including database management systems (DBMS), pattern recognition, expert systems, artificial intelligence (AI), and intelligent systems.

Fuzzy relations represent partial degrees of relations between elements in a universe of discourse and can be used to obtain consequences from a set of premises by the use of fuzzy compositional rules of inference. Some properties of fuzzy relations provide information about the consequences of the inference. For example, when an inference is performed with a reflexive and T-transitive fuzzy relation (called a T-preorder), the consequences obtained with a single fuzzy composition contain all the inferable information [11]. The consequences $C(A)$ of a fuzzy set $A$ and fuzzy inference with T-preorders are Tarski consequences, verifying fuzzy inclusion ( $A \subseteq C(A)$ ), monotony (if $A \subseteq B$ then $\mathrm{C}(A) \subseteq C(B)$ ), and idempotence $(C(C(A))=C(A))$ for any fuzzy set $A$ and $B$.

All $\alpha$-cuts of a min-transitive fuzzy relation (one of the classical relations defined by couples of elements that have a degree of relationship greater or equal to $\alpha$ ) are transitive relations [39]. This property does not hold for any t-norm other than the minimum. Only the t-norm minimum satisfies the property that the mintransitive closure operation commutes with the transitive closure of the $\alpha$-cut classical relation. These huge differences between the properties of the min $t$ norm and those of other t-norms pose a new challenge to efforts to design extensions of useful algorithms that are defined only for the min t-norm.

Analogously to transitivity of crisp relations, the transitive property of fuzzy relations can be understood as a threshold on the degree of the relation (for example, a degree of equality) between two elements, when a degree of relation between those elements and a third element of a universe of discourse is known. Either in a crisp or fuzzy framework, the chances of violating transitivity increases with the number of elicitations. Each elicitation is subject to error and each error may produce an inconsistency. In practice, this will be mostly the case when dealing even with a not very big number of elements, whenever such elicitations have to be made in a continuous range of values, as happens in the fuzzy context, where intransitivity of indifference chains in the classical sugar paradox is explained in terms of fuzzy sweetness preference). Hence, 
developing procedures to estimate the true transitive relation from intransitive elicitations become crucial in many contexts (but remind that transitivity neither completeness are the unique approaches to consistency,

Algorithms to compute T-transitive fuzzy relations are useful in many branches of artificial intelligence. Among the applications of similarities and Tindistinguishabilities are classification and clustering methods, which allow us to distinguish and to "classify" objects . Such fuzzy relations can be used to represent the concepts of equality and neighborhood, thus generalizing the classical equivalence relations. Clustering applications often need to compute fuzzy equivalence relations from reflexive and symmetric fuzzy relations.

Fuzzy relations on a finite set can also represent labeled directed graphs Symmetric fuzzy relations can represent weighted complete undirected graphs, where the set of nodes is the universe and the weights of the edges are the relationship degrees. All triangular paths of a T-transitive graph are T-transitive.

In approximate and uncertain reasoning, the $\mathrm{T}$-transitivity property is important, and sometimes the knowledge acquired is represented by a non-Ttransitive relation to be replaced by the closest possible T-transitive relation. Given a fuzzy relation $R$ (or a directed graph), its T-transitive closure $R^{\mathrm{T}}$ is the lowest T-transitive fuzzy relation that contains $R$. In the past few years, some authors have developed new, fast algorithms to compute the min-transitive closure of fuzzy relations However only a few methods exist to compute a T-transitive lower approximation of a given fuzzy relation , and in general a unique T-transitive lower approximation does not exist.

This paper proposes a fast method to compute one or several T-transitive lower approximations or T-transitive openings of a reflexive and symmetric fuzzy relation for the minimum t-norm and for strictly increasing t-norms (for example, all t-norms in the family of the product t-norm ).

A method to compute a T-transitive lower approximation of fuzzy relations

can be used to provide a new measure of the T-transitivity of fuzzy relations. It can also be used to build T-transitive fuzzy relations from a given fuzzy relation. When the initial fuzzy relation is reflexive, the algorithm generates $\mathrm{T}$ preorders that are different from the T-preorders generated by $\mathrm{T}$-transitive closure. Some T-transitivization algorithms maintain reflexivity and $\alpha$-reflexivity. However, T-transitive closure maintains reflexivity, but not $\alpha$-reflexivity. The only well-established methods for generating a T-transitive opening of a fuzzy relation and any t-norm do not preserve the symmetry property.

The T-transitive closure is uniquely defined; however, several T-transitive relations are contained in the initial relation. After generating 100 random fuzzy relations for all dimensions from 2 to 100 and computing their average distance with the T-transitive closure and with the computed T-transitive lower approximation, it has been observed that for any distance, for any t-norm, and for any dimension that the computed T-transitive lower approximation is closer to the initial relations than the T-transitive closure when using average distances.

There exist several T-transitive openings of a given fuzzy relation, but in general, the highest T-transitive opening cannot be found. , two algorithms that compute a T-transitive lower approximation from a symmetric fuzzy relation are given, but those algorithms do not always yield a T-transitive opening. A set 
of T-transitive fuzzy relations, where $\mathrm{T}$ gradually moves towards the minimum operator, has also been investigated inside a parameterized family of t-norms. Leclerc has proved that an injective and symmetric fuzzy relation on a universe of $n$ elements has exactly $(n-1)$ ! symmetric min-transitive openings.

To the authors' knowledge, the problem of finding one or several T-transitive openings of a proximity relation without losing the symmetry property has still not been solved. This paper is a step towards such a solution, by proposing the existence of a reflexive and symmetric T-transitive lower approximation or opening from a reflexive and symmetric fuzzy relation

\section{Preliminaries}

Let $E=\left\{e_{1}, \ldots, e_{\mathrm{n}}\right\}$ be a finite set.

A fuzzy relation is a map $R: E \times E \rightarrow[0,1]$ and the degree of the relation for elements $e_{i}$ and $e_{j}$ is denoted as $e_{i j}$. So $e_{i j}=R\left(e_{\mathrm{i}}, e_{\mathrm{j}}\right)$.

A fuzzy relation $R$ is reflexive if $e_{i i}=1$ for $1 \leq i \leq n$.

A fuzzy relation $R$ is symmetric if $e_{i j}=e_{j i}$ for $1 \leq i, j \leq n$.

Definition 2.1: A binary operation $\mathrm{T}:[0,1] \times[0,1] \rightarrow[0,1]$ is a t-norm $[33]$ if it satisfies the following axioms:
1. $\mathrm{T}(1, x)=x$
2. $\mathrm{T}(x, y)=\mathrm{T}(y, x)$
3. $\mathrm{T}(x, \mathrm{~T}(y, z))=\mathrm{T}(\mathrm{T}(x, y), z)$
4. If $x \leq x^{\prime}$ and $y \leq y^{\prime}$ then $\mathrm{T}(x, y) \leq \mathrm{T}\left(x^{\prime}, y^{\prime}\right)$

Definition 2.2. Let $\mathrm{T}$ be a triangular norm. A fuzzy relation $R: E \times E \rightarrow[0,1]$ is $T$-transitive if $\mathrm{T}(R(a, b), R(b, c)) \leq R(a, c)$ for all $a, b, c$ in $E$. So, $\mathrm{T}\left(e_{i k}, e_{k j}\right) \leq e_{i j}$ for all $i, j, k$ from 1 to $n$.

Definition 2.3. A reflexive and symmetric fuzzy relation is called a proximity relation.

A similarity is a reflexive, symmetric and min-transitive fuzzy relation

A T-indistinguishability is a reflexive, symmetric, and T-transitive fuzzy relation

Note that a similarity is a min-indistinguishability.

Definition 2.4. A relation $A$ includes a relation $B$ (and it is denoted $A \supseteq B$ ) if $a_{i j} \geq b_{i j}$ for all $i, j$.

Definition 2.5. Let $P$ be a property of fuzzy relations on a universe $E$. A fuzzy relation $R^{P}$ is called the $P$-closture of a fuzzy relation $R$ if:

1) $R^{P}$ has property $P$

2) $R \subseteq R^{P}$

3) If $R \subseteq R^{\prime}$ and $R^{\prime}$ has property $P$, then $R^{P} \subseteq R^{\prime}$.

Theorem 2.1. $\quad$ A P-closure exists for all fuzzy relations on a universe $E$ if and only if

1) The universal relation $U_{E}$ has property $P$; 
2) The intersection of any non-empty family of fuzzy relations on Everifying property $P$, also has property $P$.

Definition 2.6. Given a t-norm $\mathrm{T}$ and a proximity relation $R$ on a finite universe, then there exists a unique T-indistinguishability $R^{\mathrm{T}}$, called the $T$ transitive closure of $R$, that includes $R$, and if a T-indistinguishability includes $R$, then it also includes $R^{\mathrm{T}}$.

Definition 2.7. Given a t-norm $\mathrm{T}$ and a fuzzy relation $R$ on a finite universe, a T-transitive lower approximation of $R$ is a T-transitive fuzzy relation included in $R[12]$.

Definition 2.8. $\quad$ Let $P$ be a property of fuzzy relations on a universe $E$. A fuzzy relation $R_{P}$ is called the $P$-opening of a fuzzy relation $R$ if:

1) $R_{P}$ has property $P$

2) $R_{P} \subseteq R$

3) If $R^{\prime}$ has property $P$ and $R_{P} \subseteq R^{\prime} \subseteq R$ then $R_{P}=R^{\prime}$.

Note that, according to this definition, a fuzzy relation $R$ can have an infinite number of $P$-openings, even on a finite universe.

In particular:

Given a t-norm $\mathrm{T}$ and a fuzzy relation $R$ on a finite universe, a $T$-transitive opening of $R$ is a relation $R_{\mathrm{T}}$ satisfying:

1) $R_{\mathrm{T}}$ is a T-transitive fuzzy relation

2) $R_{\mathrm{T}}$ is included in $R\left(R_{\mathrm{T}} \subseteq R\right)$

3) If any T-transitive relation $H$ includes $R_{\mathrm{T}}$ and is included in $R$, then it is $R_{\mathrm{T}}$ (If $\exists H \mathrm{~T}$-transitive; $R_{\mathrm{T}} \subseteq H \subseteq R$ then $H=R_{\mathrm{T}}$ ).

Note that there can be several T-transitive openings of a fuzzy relation.

Definition 2.9. The residual implication $J^{\mathrm{T}}$ of a left-continuous tnorm $\mathrm{T}$, or a quasi-inverse of $\mathrm{T}$, is the binary operation $J^{\mathrm{T}}:[0,1]^{2} \rightarrow[0,1]$, defined by $J^{\mathrm{T}}(\mathrm{x}, \mathrm{y})=\operatorname{Sup}\{\mathrm{z} \in[0,1] ; \mathrm{T}(\mathrm{x}, \mathrm{z}) \leq \mathrm{y}\}$.

In particular:

$$
\begin{gathered}
J^{\text {Min }}(x, y)= \begin{cases}1, & \text { if } x \leq y, \\
y, & \text { if } x>y\end{cases} \\
J^{\text {Prod }}(x, y)= \begin{cases}1, & \text { if } x \leq y, \\
\frac{y}{x}, & \text { if } x>y\end{cases} \\
J^{W}(x, y)=\min \{1-x+y, 1\} .
\end{gathered}
$$

For a left-continuous t-norm $\mathrm{T}$ and any $x, y \in[0,1]$, it is known that $\mathrm{T}\left(x, J^{\mathrm{T}}(x\right.$, $y)$ ) $\leq y$, i.e. $J^{\mathrm{T}}(x, y)$ is the greatest solution $z$ of the inequality $\mathrm{T}(x, z) \leq y$. In the case of a left-continuous t-norm $\mathrm{T}$ and $y \leq x$, then $J^{\mathrm{T}}(x, y)$ is the greatest solution $z$ of the equation $\mathrm{T}(x, z)=y$. 


\section{Algorithm to compute a T-transitive lower approximation or opening of a proximity relation}

\section{Algorithm 1.}

Let $A$ be a proximity relation defined on a universe of dimension $n$, let $\mathrm{T}$ be a left-continuous t-norm, and let $J^{\mathrm{T}}$ be the corresponding residual implication.

Input: a proximity relation $A$

Output: a T-indistinguisability $B$

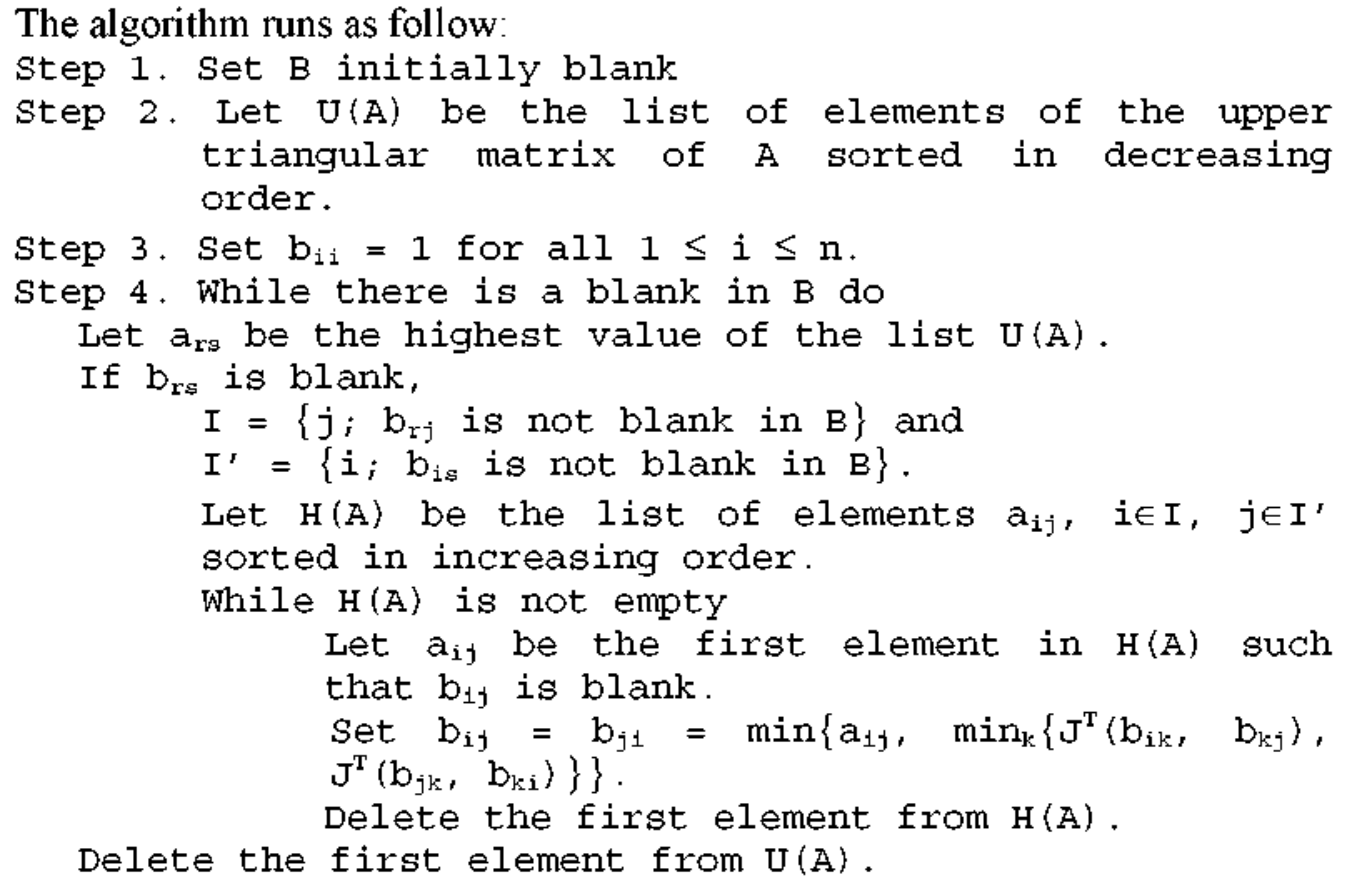

Notice in the above algorithm that the lists $U(A)$ and $H(A)$ inside the loop in step 4 are not always unique. Sometimes the highest values are repeated, and one edge must be arbitrarily chosen as the highest. However, for a given list, the algorithm provides a unique T-transitive lower approximation. Choosing a different edge with the same highest value can lead to the same T-transitive lower approximation or to a different T-transitive lower approximation. Therefore, the algorithm can also be used to find a set of T-transitive lower approximations of a proximity relation, but it always finds one T-transitive lower approximation for every possible list.

This algorithm can be regarded as a clustering algorithm. In every step, two already-obtained clusters for which there exists a linking edge that presently carries the highest weight are merged into one cluster. In the merging process, the weights of all edges between the two clusters are adjusted in a precise order (starting from the link that carries the lowest weight). By analyzing the algorithm from this perspective, it is clear that it always generates a $\mathrm{T}$-indistinguishability that lies entirely below the given fuzzy relation. 
Example 1. Given the following proximity relation:

$$
A=\left(\begin{array}{cccc}
1 & 0.7 & 0.8 & 0.9 \\
0.7 & 1 & 0.2 & 0.3 \\
0.8 & 0.2 & 1 & 0.7 \\
0.9 & 0.3 & 0.7 & 1
\end{array}\right)
$$

the algorithm can be applied as follows to compute a min-transitive lower approximation or a min-transitive opening $B$.

Step 1: Set $B$ to be blank

Step 2: Let $U(A)$ be the list of elements of the upper triangular matrix of $A$ sorted in decreasing order: $U(A)=\left(a_{14}, a_{13}, a_{34}, a_{12}, a_{24}, a_{23}\right)=(0.9,0.8,0.7,0.7$, $0.3,0.2$ ).

Step 3: Set $b_{i i}=1$ for all $i$.

$$
B=\left(\begin{array}{llll}
1 & & & \\
& 1 & & \\
& & 1 & \\
& & & 1
\end{array}\right) .
$$

Step 4: The greatest value of $U(A), a_{14}=0.9$, is chosen. Let $I=\left\{j ; b_{1 j}\right.$ that are not blank values in matrix $B\}=\{1\}$ and let $I^{\prime}=\left\{i ; b_{i 4}\right.$ that are not blank in matrix $B\}=\{4\} . H(A)=\left(a_{14}\right)$. The values $b_{41}=b_{14}=a_{14}=0.9$ are inserted into $B$.

$$
B=\left(\begin{array}{cccc}
1 & & & 0.9 \\
& 1 & & \\
& & 1 & \\
0.9 & & & 1
\end{array}\right) \text {. }
$$

The next greatest element in $U(A)$ is $a_{13}=0.8 . I=\left\{j ; b_{1\}}\right.$ are not blank in $\left.B\right\}=$ $\{1,4\}$ and $I^{\prime}=\left\{i ; b_{i 3}\right.$ is not blank in $\left.B\right\}=\{3\}$. The values $b_{13}, b_{34}$, and their symmetric values are inserted into $B . H(A)=\left(a_{34}, a_{13}\right)=(0.7,0.8)$, then $b_{43}=b_{34}=$ $a_{34}=0.7$ and $b_{13}=b_{31}=\min \left\{a_{13}, \min _{k}\left\{J^{\mathrm{T}}\left(b_{1 k}, b_{k 3}\right), J^{\mathrm{T}}\left(b_{3 k}, b_{k 1}\right)\right\}\right\}=\min \left\{a_{13}\right.$, $\left.\min \left\{J^{\mathrm{T}}\left(b_{14}, b_{43}\right), J^{\mathrm{T}}\left(b_{34}, b_{41}\right)\right\}\right\}=\min \left\{0.8, \min \left\{J^{\mathrm{Min}}(0.9,0.7), J^{\mathrm{Min}}(0.7,0.9)\right\}\right\}=$ $\min \{0.8, \min \{0.7,1\}\}=0.7$.

$$
B=\left(\begin{array}{cccc}
1 & & 0.7 & 0.9 \\
& 1 & & \\
0.7 & & 1 & 0.7 \\
0.9 & & 0.7 & 1
\end{array}\right)
$$

The next greatest element in $U(A)$ is $a_{12}=0.7 . I=\left\{j ; b_{1 j}\right.$ are not blank in $\left.B\right\}=$ $\{1,3,4\}$ and $I^{\prime}=\left\{i ; b_{i 2}\right.$ is not blank in $\left.B\right\}=\{2\} . H(A)=\left(a_{23}, a_{24}, a_{12}\right)=(0.2,0.3$, 
$0.7)$. The values $b_{23}=b_{32}=a_{23}=0.2 ; b_{24}=b_{42}=\min \left\{a_{24}, \min _{k}\left\{J^{\mathrm{T}}\left(b_{4 k}, b_{k 2}\right), J^{\mathrm{T}}\left(b_{2 k}\right.\right.\right.$, $\left.\left.\left.b_{k 4}\right)\right\}\right\}=\min \left\{a_{24}, \min \left\{J^{\mathrm{T}}\left(b_{43}, b_{32}\right), J^{\mathrm{T}}\left(b_{23}, b_{34}\right)\right\}\right\}=\min \left\{0.3, \min \left\{J^{\mathrm{Min}}(0.7,0.2)\right.\right.$, $\left.\left.J^{\mathrm{Min}}(0.2,0.7)\right\}\right\}=\min \{0.3, \min \{0.2,1\}\}=0.2 ; b_{12}=b_{21}=\min \left\{a_{12}, \min _{k}\left\{J^{\mathrm{T}}\left(b_{1 k}\right.\right.\right.$ $\left.\left.\left.b_{k 2}\right), J^{\mathrm{T}}\left(b_{2 k}, b_{k 1}\right)\right\}\right\}=\min \left\{a_{12}, \min \left\{J^{\mathrm{T}}\left(b_{13}, b_{32}\right), J^{\mathrm{T}}\left(b_{23}, b_{31}\right), J^{\mathrm{T}}\left(b_{14}, b_{42}\right), J^{\mathrm{T}}\left(b_{24}\right.\right.\right.$, $\left.\left.\left.b_{41}\right)\right\}\right\}=\min \left\{0.7, \min \left\{J^{\mathrm{Min}}(0.7,0.2), J^{\mathrm{Min}}(0.2,0.7), J^{\mathrm{Min}}(0.9,0.2), J^{\mathrm{Min}}(0.2,0.9)\right\}\right\}$ $=\min \{0.7, \min \{0.2,1,0.2,1\}\}=0.2$.

$$
B=\left(\begin{array}{cccc}
1 & 0.2 & 0.7 & 0.9 \\
0.2 & 1 & 0.2 & 0.2 \\
0.7 & 0.2 & 1 & 0.7 \\
0.9 & 0.2 & 0.7 & 1
\end{array}\right)
$$

A Prod-transitive lower approximation given by this method is:

$$
B=\left(\begin{array}{cccc}
1 & 0.257 & 0.777 & 0.9 \\
0.257 & 1 & 0.2 & 0.285 \\
0.777 & 0.2 & 1 & 0.7 \\
0.9 & 0.285 & 0.7 & 1
\end{array}\right)
$$

The steps to reach $B$ are computed as follows:

Set $b_{i j}=1$ for all $i$.

$$
b_{41}=b_{14}=a_{14}=0.9
$$

$b_{43}=b_{34}=a_{34}=0.7 ; b_{13}=b_{31}=\min \left\{a_{13}, \min _{k}\left\{J^{\mathrm{T}}\left(b_{1 k}, b_{k 3}\right), J^{\mathrm{T}}\left(b_{3 k}, b_{k 1}\right)\right\}\right\}=$ $\min \left\{a_{13}, \min \left\{J^{\mathrm{T}}\left(b_{14}, b_{43}\right), J^{\mathrm{T}}\left(b_{34}, b_{41}\right)\right\}\right\}=\min \left\{0.8, \min \left\{J^{\mathrm{Prod}}(0.9,0.7), J^{\operatorname{Prod}}(0.7\right.\right.$, $0.9)\}\}=\min \{0.8, \min \{0.7 / 0.9,1\}\}=0.777$

$b_{23}=b_{32}=a_{23}=0.2 ; b_{42}=b_{24}=\min \left\{a_{24}, \min _{k}\left\{J^{\mathrm{T}}\left(b_{4 k}, b_{k 2}\right), J^{\mathrm{T}}\left(b_{2 k}, b_{k 4}\right)\right\}\right\}=$ $\min \left\{a_{24}, \min \left\{J^{\mathrm{T}}\left(b_{43}, b_{32}\right), J^{\mathrm{T}}\left(b_{23}, b_{34}\right)\right\}\right\}=\min \left\{0.3, \min \left\{J^{\text {Prod }}(0.7,0.2), J^{\text {Prod }^{2}}(0.2\right.\right.$, $0.7)\}\}=\min \{0.3, \min \{0.2 / 0.7,1\}\}=0.285$;

$b_{12}=b_{21}=\min \left\{a_{12}, \min _{k}\left\{J^{\mathrm{T}}\left(b_{1 k}, b_{k 2}\right), J^{\mathrm{T}}\left(b_{2 k}, b_{k 1}\right)\right\}\right\}=\min \left\{a_{12}, \min \left\{J^{\mathrm{T}}\left(b_{13}\right.\right.\right.$, $\left.\left.\left.b_{32}\right), J^{\mathrm{T}}\left(b_{23}, b_{31}\right), J^{\mathrm{T}}\left(b_{14}, b_{42}\right), J^{\mathrm{T}}\left(b_{24}, b_{41}\right)\right\}\right\}=\min \left\{0.7, \min \left\{J^{\mathrm{Prod}}(0.777,0.2)\right.\right.$, $\left.\left.J^{\text {Prod }}(0.2,0.777), J^{\text {Prod }}(0.9,0.285), J^{\text {Prod }}(0.285,0.9)\right\}\right\}=\min \{0.7, \min \{0.2 / 0.777,1$, $0.285 / 0.9,1\}\}=\min \{0.7,0.257,1,0.316\}=0.257$.

A W-transitive lower approximation given by this method is:

$$
B=\left(\begin{array}{cccc}
1 & 0.4 & 0.6 & 0.9 \\
0.4 & 1 & 0.2 & 0.3 \\
0.6 & 0.2 & 1 & 0.7 \\
0.9 & 0.3 & 0.7 & 1
\end{array}\right)
$$

The steps to obtain $B$ are computed as follows:

Set $b_{i j}=1$ for all $i$.

$$
b_{41}=b_{14}=a_{14}=0.9 \text { and } \alpha=0.9 \text {. }
$$


$b_{43}=b_{34}=a_{34}=0.7 ; b_{13}=b_{31}=\min \left\{a_{13}, \min _{k}\left\{J^{\mathrm{T}}\left(b_{1 k}, b_{k 3}\right), J^{\mathrm{T}}\left(b_{3 k}, b_{k 1}\right)\right\}\right\}=$ $\min \left\{a_{13}, \min _{k}\left\{J^{\mathrm{T}}\left(b_{14}, b_{43}\right), J^{\mathrm{T}}\left(b_{34}, b_{41}\right)\right\}\right\}=\min \left\{0.8,0.9, \min \left\{J^{W}(0.9,0.7), J^{W}(0.7\right.\right.$, $0.9)\}\}=\min \{0.8,0.9, \min \{0.6,1\}\}=0.6$

$b_{23}=b_{32}=a_{23}=0.2 ; b_{42}=b_{24}=\min \left\{a_{24}, \min _{k}\left\{J^{\mathrm{T}}\left(b_{4 k}, b_{k 2}\right), J^{\mathrm{T}}\left(b_{2 k}, b_{k 4}\right)\right\}\right\}=$ $\min \left\{a_{24}, \min \left\{J^{\mathrm{T}}\left(b_{43}, b_{32}\right), J^{\mathrm{T}}\left(b_{23}, b_{34}\right)\right\}\right\}=\min \left\{0.3, \min \left\{J^{\mathrm{W}}(0.7,0.2), J^{\mathrm{W}}(0.2\right.\right.$, $0.7)\}\}=\min \{0.3, \min \{0.5,1\}\}=0.3$.

$b_{12}=b_{21}=\min \left\{a_{12}, \min _{k}\left\{J^{\mathrm{T}}\left(b_{1 k}, b_{k 2}\right), J^{\mathrm{T}}\left(b_{2 k}, b_{k 1}\right)\right\}\right\}=\min \left\{a_{12}, \min \left\{J^{\mathrm{T}}\left(b_{13}\right.\right.\right.$, $\left.\left.\left.b_{32}\right), J^{\mathrm{T}}\left(b_{23}, b_{31}\right), J^{\mathrm{T}}\left(b_{14}, b_{42}\right), J^{\mathrm{T}}\left(b_{24}, b_{41}\right)\right\}\right\}=\min \left\{0.7, \min \left\{J^{\mathrm{W}}(0.6,0.2), J^{\mathrm{W}}(0.2\right.\right.$, $\left.\left.0.6), J^{W}(0.9,0.3), J^{W}(0.3,0.9)\right\}\right\}=\min \{0.7, \min \{0.6,1,0.4,1\}\}=0.4$.

Lemma 1. The output of the algorithm applied to a proximity relation $A$ is a T-indistinguishability $B$, such that $B \subseteq A$.

Proof: By the construction method, $B$ is a reflexive fuzzy relation (step 3 ), $B$ is a symmetric fuzzy relation ( $\operatorname{step} 4: b_{i j}=b_{j i}=\ldots$ ), and $B \subseteq A$ because for all $i, j, b_{i j}$ $=\min \left\{a_{i j}, \ldots\right\} \leq a_{i j}$.

The T-transitive property of $B$ is imposed by the assignment $b_{i j}=b_{j i}=\min \left\{a_{i j}\right.$, $\left.\min _{k}\left\{J^{\mathrm{T}}\left(b_{i k}, b_{k j}\right), J^{\mathrm{T}}\left(b_{j k}, b_{k g}\right)\right\}\right\}$. Then $b_{i j} \leq \min _{k}\left\{J^{\mathrm{T}}\left(b_{t k}, b_{k j}\right), J^{\mathrm{T}}\left(b_{j k}, b_{k i}\right)\right\}$ and if $b_{i k}$ $>b_{k j}$ then $b_{i j} \leq \min _{k}\left\{J^{\mathrm{T}}\left(b_{i k}, b_{k j}\right)\right\}$ and then $b_{i k} \geq \mathrm{T}\left(b_{i j}, b_{k j}\right)=\mathrm{T}\left(b_{i j}, b_{j k}\right)$ for all $j$.

The following lemma shows that the previous algorithm gives a T-transitive lower approximation of a reflexive and symmetric fuzzy relation when $\mathrm{T}$ is the minimum t-norm or a strictly increasing t-norm (for example, the family of all product t-norms).

Lemma 2. The T-transitive lower approximation of a proximity relation computed by algorithm 1 takes $O\left(n^{3}\right)$ time complexity in the worst case.

Proof. The computational time complexity of the algorithm can be analyzed as follows:

Step 2 sorts $\frac{n^{2}}{2}-n$ values, so it takes $O\left(n^{2} \log n\right)$ time complexity.

Step 4: A general proof of the time complexity of step four can be achieved by computing the complexity in the worst case, which is the case in which $n-1$ blocks of dimension one must be filled. Suppose that for each value in $U(A)$, a block of dimension one must be added. Then $n-1$ blocks must be constructed in $B$, numbered from $k=2$ to $n$, and each block-construction step must sort the $(k-1)$ elements in $H(A)$ and then make the $k-1$ assignments in $B$. Then the final time complexity of the step four is of the order of $\sum_{k=2}^{n}$ ((sort of $k$-1 elements in $\left.H(A)\right)+$ (assignments of $k-1$ elements in $B$, looking at the previous assignments)). The step four time complexity can therefore be computed as follows: 


$$
\mathrm{O}\left(\sum_{k=2}^{n}\left(\left(\sum_{j=1}^{k-1} j\right)\right)=\mathrm{O}\left(\sum_{k=2}^{n}\left(\frac{(k-1)+1}{2}(k-1)\right)\right)=\mathrm{O}\left(\sum_{k=2}^{n}\left(k^{2}\right)\right)=\mathrm{O}\left(n^{3}\right)\right.
$$

which is the time complexity of the algorithm.

Note that the most efficient known algorithm for computing the T-transitive closure of a fuzzy relation, with $\mathrm{T}$ other that the minimum $\mathrm{t}$-norms, also takes $\mathrm{O}\left(n^{3}\right)$ time.

\section{Conclusions}

An $\mathrm{O}\left(n^{3}\right)$ time algorithm to compute a T-transitive lower approximation or opening of a proximity relation (a reflexive and symmetric fuzzy relation) has been given.

Sometimes the algorithm must choose an edge from the (possibly repeated) highest value of an ordered list. For every chosen list, the algorithm computes a Ttransitive approximation. Therefore, in some cases, the algorithm can be used to find several T-transitive approximations or openings.

It remains an open problem whether the algorithm gives a T-transitive opening for all continuous t-norms.

It is also proved that the algorithm preserves the reflexivity and symmetry properties and so, the computed T-transitive lower approximation or opening is a T-indistinguishability.

Applications of this algorithm can be considered in various fields, including deductive databases, pattern recognition, expert systems, artificial intelligence and intelligent systems.

An implementation in java of the proposed algorithm for the minimum, product and Lukasiewicz t-norms can be downloaded from http:/www.fdi.ucm es/profesor/lgarmend/SC/Programas/openings.jar

To run it it is necessary to install a 'java virtual machine' or 'Java Runtime Environment' (JDK), downloadable at http:/java.sun.com/. Most computers already have it installed.

\section{Acknowledgements}

This research is partially supported by the Spanish Ministry of Science and Technology, grant number TIN2006-06190, the Research Group 910149 at Complutense University of Madrid and the Research Group MAIC at Technical University of Madrid.

\section{References}

C. Alsina, E. Trillas, L. Valverde, On some logical connectives for fuzzy set theory, J. Math. Ann. Appl. 93 (1983) 15-26.

W. Bandler, J. Kohout, Special properties, closures and interiors of crisp and fuzzy relations, Fuzzy Sets and Systems 26 (1988) 317-331. 
D. Boixader, On the relationship between T-transitivity and approximate equality, Fuzzy Sets and Systems 33 (2003) 6-69.

V. Cutello, J. Montero, Fuzzy rationality measures. Fuzzy Sets and Systems 62 (1994) 39-44.

P. Dawyndt, H. De Meyer, B. De Baets, The complete linkage clustering algorithm revisited, Soft Computing 9 (2005) 385-392.

P. Dawyndt, H. De Meyer, B. De Baets, UPGMA clustering revisited: a weight-driven approach to transitive approximation, Internat. $\mathrm{J}$. Approximate Reasoning 42 (2006) 174-191.

B. De Baets, H. De Meyer, Transitive approximation of fuzzy relations by alternating closures and openings, Soft Computing 7 (2003) 210-219

B. De Baets, H. De Meyer, On the existence and construction of Ttransitive closures, Inform. Sci. 152 (2003) 167-179.

A. Di Nola, W. Kolodziejczyk, S. Sessa, Transitive solutions of relational equations on finite sets and linear lattices, Lecture Notes in Computer Science, 521 Springer, Berlin, 1991, pp. 173-182.

D. Dubois, H. Prade, Fuzzy Sets and Systems: Theory and Applications, Academic Press, New York, 1980.

J. Elorza, P. Burillo, On the relation of fuzzy preorders and fuzzy consequence operators, International Journal of Uncertainty, Fuzziness and Knowledge-based Systems 7 (3) (1999).

F. Esteva, P. Garcia, L. Godo, R. O. Rodriguez, Fuzzy approximation relations, modal structures and possibilistic logic, Mathware and Soft Computing 5 (2-3) (1998) 151-166.

J. Fodor, M. Roubens, Structure of transitive valued binary relations, Math. Soc. Sci. 30 71-94 (1995).

L. Garmendia, C. Campo, S. Cubillo, A. Salvador, A Method to Make Some Fuzzy Relations T-Transitive, International Journal of Intelligence Systems. 14, 9, (1999) $873-882$.

L. Garmendia, A. Salvador, On a new method to T-transitivize fuzzy relations, in B. Bouchon-Meunier, J. Gutierrez-Rios, L. Magdalena, R. R. Yager, (eds.), Technologies for Constructing Intelligent Systems 2, Springer. 2000 , pp. $251-260$.

L. Garmendia; A. Salvador. A new algorithm to compute low TTransitive approximation of a fuzzy relation preserving symmetry. Comparisons with the T-transitive closure. Springer-Verlag Lecture Notes in Artificial Intelligence. European Conferences on Symbolic and Quantitative Approaches to Reasoning with uncertainty, (2005) 576-586.

L. Garmendia; A. Salvador. Computing a transitive opening of a reflexive and symmetric fuzzy relation. Springer-Verlag Lecture Notes in Artificial Intelligence, European Conferences on Symbolic and Quantitative Approaches to Reasoning with uncertainty (2005) 587-599.

H. Hashimoto, Transitivity of generalised fuzzy matrices, Fuzzy Sets and Systems. 17, 1, (1985) 83-90.

J. Jacas, On the generators of T-indistinguishability operators, Stochastica 12 (1988) 49-63.

J. Jacas, Similarity relations. The calculation of minimal generating families, Fuzzy Sets and Systems 35 (1990) 151-162 
J. Jacas, J. Recasens, Fuzzy T-transitive relations: eigenvectors and generators, Fuzzy Sets and Systems 72 (1995) 147-154

J. Jacas, J. Recasens, Decomposable indistinguishability operators, Proceedings of the Sixth IFSA Congress, Sao Paulo, 1995.

G. J. Klir, B. Yuan, Fuzzy Sets and Fuzzy Logic. Theory and Applications, Prentice Hall, New Jersey, 1995.

B. Leclerc, Caractérisation construction et dénombrement des ultramétriques supérieures minimales, Statistique et Analyse des Donées 11 (1986), 26-50

H.-S. Lee, An optimal algorithm for computing the max-min transitive closure of a fuzzy similarity matrix, Fuzzy Sets and Systems 123 (2001) 129-136.

F. Montero, J., Tejada, Some problems on the definition of fuzzy preference relation, Fuzzy Sets and Systems 20 (1986) 45-53.

J. Montero, Arrow's theorem under fuzzy rationality, Behavioral Science 32 (1987) 267-273 (now Systems Research and Behavioral Science)

J. Montero, D. Gómez, J. Yánez, J. Gonález-Pachón, Rationality cores in preference representation. Proceedings International Fuzzy Systems Association Conference, Bogaziai University, Istanbul (2003) 340-343.

H. Naessens, H. De Meyer, B. De Baets, Algorithms for the Computation of T-Transitive Closures, IEEE Trans Fuzzy Systems 104 (2002) 541-551

S. Ovchinnikov, Representations of Transitive Fuzzy Relations, in Aspects of Vagueness, H. J. Skala, S. Termini and E. Trillas (eds.), Reidel Pubs. (1984) 105-118.

H. B. Potoczny, On similarity relations in fuzzy relational databases, Fuzzy Sets and Systems 12 (3) (1984) 231-235

R. O. Rodriguez, F. Esteva, P. Garcia, L. Godo, L., On implicative closure operators in approximate reasoning, International Journal of Approximate Reasoning 33 (2003) 159-184.

B. Schweizer, A. Sklar, Probabilistic Metric Spaces, North-Holland, New York, 1983.

E. Trillas, L. Valverde, An inquiry into indistinguishability operators, in Aspects of Vagueness, H. J. Skala, S. Termini y E. Trillas (eds.), Reidel Pubs. (1984) 231-256.

L. Valverde, On the structure of F-indistinguishability operators, Fuzzy Sets and Systems 17 (1985) 313-328.

M. Wagenknecht, On transitive solutions of fuzzy equations, inequalities and lower approximation of fuzzy relations, Fuzzy Sets and Systems 75 (1995) 229-240.

M. Wallace, Y. Avrithis, S. Kollias, Computationallly efficient sup-t transitive closure for sparse fuzzy binary relations, Fuzzy Sets and Systems 157 (3) (2006) 341-372.

Xian Xiao, An algorithm for calculating fuzzy transitive closure, Fuzzy Math. 5 (4) (1985) 71-73.

L. A. Zadeh, Fuzzy sets, Inform. \& Control 8 (1965) 338-353. 
L. A. Zadeh, Similarity relations and fuzzy orderings, Inform. Sci. 3 (1971) 177-200. 